ÉGYPTE

monde arabe

\section{Égypte/Monde arabe}

26 | 1996

Mutations

\title{
De la langue et en deçà : nationalismes arabes à géométrie variable
}

\section{Eberhard Kienle}

\section{(2) OpenEdition}

\section{Journals}

Édition électronique

URL : https://journals.openedition.org/ema/1478

DOI : 10.4000/ema.1478

ISSN : 2090-7273

\section{Éditeur}

CEDEJ - Centre d'études et de documentation économiques juridiques et sociales

\section{Édition imprimée}

Date de publication : 30 juin 1996

Pagination : 153-170

ISSN : 1110-5097

\section{Référence électronique}

Eberhard Kienle, «De la langue et en deçà : nationalismes arabes à géométrie variable », Égypte/Monde arabe [En ligne], 26 | 1996, mis en ligne le 08 juillet 2008, consulté le 07 juillet 2022. URL : http:// journals.openedition.org/ema/1478; DOI : https://doi.org/10.4000/ema.1478

Ce document a été généré automatiquement le 7 juillet 2022.

Tous droits réservés 


\title{
De la langue et en deçà : nationalismes arabes à géométrie variable
}

\author{
Eberhard Kienle
}

\section{NOTE DE L'ÉDITEUR}

Communication présentée au colloque « Langues et pouvoir » à l'occasion du bicentenaire de l'Inalco, Paris, 11 au 13/10/95.

1 Depuis que les mouvements islamistes monopolisent l'intérêt, l'étude des phénomènes nationalistes dans le monde arabe n'a plus guère droit de cité dans les sciences sociales ${ }^{1}$. Conséquence ou non de cette mutation, les pays arabes semblent à l'abri du regain des fièvres nationalistes qui surgissent un peu partout dans le monde depuis la fin de la guerre froide. Si l'on voit advenir un "nouvel âge du nationalisme», la «délaïcisation» du politique paraît d'ores et déjà en exclure les pays arabes. L'on pourrait tout au plus voir dans l'islamisme une sorte d'Aufhebung ou de « dépassement » hégélien du nationalisme, comme certains l'ont noté lors de la guerre du Golfe en 1990-91. Il n'est donc pas surprenant que la "thématisation» des mouvements et idéologies nationalistes dans les pays arabes n'ait plus guère d'attrait sur les esprits. Ainsi est-il de bon ton d'affirmer que Jacques Berque avait surestimé la force du nationalisme arabe.

2 Le fait que les phénomènes nationalistes dans les pays arabes n'aient guère été au centre des préoccupations des chercheurs ces derniers temps se perçoit par exemple dans l'utilisation quasi exclusive de la notion de «nationalisme arabe » au singulier. Non seulement il y a identification des phénomènes nationalistes dans le monde arabe avec la notion de "nationalisme arabe ", mais également avec un nationalisme arabe unifié et indivisible. En revanche, florissant à l'époque des vérités multiples, les études 
sur les phénomènes «islamiques » ou «islamistes" parlent tout naturellement $\mathrm{d}^{\prime}$ ' islams » ou d'« islamismes » au pluriel ${ }^{2}$.

3 Certes, il est désormais acquis que «le» nationalisme arabe s'est manifesté de différentes manières dans le temps et dans l'espace. Ainsi distingue-t-on le nationalisme conservateur ou «libéral » des classes aisées sous le mandat, qui avait pour but principal l'indépendance politique, et le nationalisme « révolutionnaire » et socialisant des nouvelles forces politiques d'origine sociale plus modeste qui prirent le pouvoir au cours des années cinquante et soixante ${ }^{3}$. À en croire les ba'thistes qui, pendant ces deux décennies, s'imposèrent en Syrie et en Irak, leur nationalisme innovait, porteur d'un projet social et économique devant servir les intérêts de tous les membres de la "nation"; de par cet objectif et la destruction des anciennes classes aisées qu'il impliquait, ce nationalisme se prétendait également plus militant, plus fort et plus efficace ${ }^{4}$.

4 Quand au nationalisme conservateur ou «libéral » représenté par ces classes aisées, devenues dominantes avec l'indépendance, on y distingue couramment les tendances républicaines de la majorité du Parti national (al-hizb al-watani) en Syrie et les tendances monarchistes comme on en trouvait surtout au sein de son concurrent, le Parti du peuple (hizb al-sha'b), et bien sûr parmi les acolytes des dynasties hachémites en Irak et Jordanie ${ }^{5}$. On répète non moins couramment que le nationalisme « révolutionnaire » des ba'thistes différait de celui des nassériens, même après que ces derniers eurent abandonné leur rhétorique égypto-centriste ${ }^{6}$. Enfin, on s'est efforcé d'identifier en détail les différences opposant le balhisme des pères fondateurs et celui de leurs disciples et successeurs, comme le montrerait la comparaison entre la "constitution" (dustur)du parti de 1947 et les "points de départ» (muntalaqât nazariyya) énoncés en $1963^{7}$.

5 Pourtant, les différences ainsi établies apparaissent le plus souvent comme des différences accidentelles de réalités essentiellement identiques : il s'agirait, plutôt que de « nationalismes arabes », de diverses variantes « $d u$ » nationalisme arabe. Le passage du singulier au pluriel reste donc en suspens. Vu cette conception arborescente, sinon monolithique, qui ramène tout au même "isme", il n'est pas surprenant que les mouvements nationalistes qui, dans le monde arabe, ne sont pas des "nationalismes arabes» restent à l'ombre « $d u$ » nationalisme arabe. Ces mouvements et leurs influence n'ont été analysés de façon systématique et approfondie qu'en ce qui concerne l'époque de leur formation ${ }^{8}$. La notion de «nation égyptienne » qui dominait le discours de la monarchie, du Wafd et des autres acteurs politiques de l'époque libérale ${ }^{9}$, ne fut-elle pas abandonnée quelques années après la révolution de 1952 au profit de la notion de «nation arabe »? Et l'évolution du Parti populaire syrien (al-hizb al-qawmi al-sûri) ne confirme-t-elle pas cette même tendance? Chantre d'un nationalisme syrien au départ, il redéfinissait, au cours des années cinquante, les frontières de la Syrie en y incluant tout le Machrek et même l'île de Chypre ${ }^{10}$.

6 Malgré ces processus qui conduisirent à l'« arabisation » de l'Égypte et, bien davantage, de la Syrie, des notions d'identités collectives moins vastes subsistaient dans les deux pays. Le plus souvent, ces identités collectives sont considérées comme des facteurs de division interne des populations, parfois au point qu'il serait impossible de les considérer comme des "sociétés " religieuses, ethniques, tribales ou de 'asabiyya continuent à faire couler beaucoup $\mathrm{d}^{\prime}$ encre $^{12}$ tandis que les identités «horizontales», de classe, ont été de plus en plus 
négligées au cours des années quatre-vingt, parfois sous prétexte qu'elles étaient peu influentes à l'époque des fondamentalismes ${ }^{13}$. Mais si l'analyse des phénomènes politiques en termes de classe ou, du moins, d'identités de classe a eu ses heures de gloire et connaît même une renaissance certaine ${ }^{14}$, l'importance d'identités collectives qui coïncident ou sont construites afin de coïncider avec les frontières d'États arabes individuels n'a jamais été suffisamment mise en relief ${ }^{15}$.

7 Pourtant, l'exemple de l'Égypte surtout, et même celui de la Syrie, montrent que les identités collectives qui se définissent par rapport aux États ont, jusqu'à aujourd'hui, concurrencé les nationalismes arabes ou coexisté avec eux. S'adressant à la population entière d'un territoire clairement défini, ces formes d'identité collective sont des identités nationales si les groupes ainsi constitués réussissent à obtenir la solidarité et la loyauté suprême de leurs membres. Dans la mesure où ces identités sont produites et reproduites sur la base d'une construction idéologique, elles constituent des nationalismes ${ }^{16}$.

8 Le but de la présente contribution n'est évidemment pas de répéter ou résumer les arguments qui, malgré certaines discontinuités, continuent d'animer les débats arabes sur les rapports entre les deux notions de wataniyya et qawmiyya, la première désignant précisément ces nationalismes restreints aux États tandis que la seconde est réservée aux nationalismes arabes ou $"$ pan-arabes $»^{17}$. Ce qui, dans le contexte actuel, nous intéresse davantage, ce sont les conditions qui favorisent l'une ou l'autre conception de la nation: celle - qawmi- qui le plus souvent repose sur la langue arabe et, par conséquent, inclut tous ceux qui la parlent, et celle - watani - qui n'est jamais basée sur la langue. Cette problématique sera abordée à travers la comparaison des fortunes qu'ont connues ces deux notions en Syrie et en Égypte après la deuxième guerre mondiale.

\section{Arabisation et désarabisation de la nation en Syrie et en Égypte}

9 Les cas syrien et égyptien se prêtent particulièrement à une telle comparaison parce qu'au-delà des ressemblances nécessaires pour entreprendre une telle démarche, ils présentent également des différences qui la rendent significative. Comme nous l'avons suggéré plus haut, l'importance et l'influence des deux notions de nation - l'une large, basée sur la continuité linguistique, l'autre étroite, basée sur les discontinuités politiques - n'ont pas été les mêmes dans les deux pays. Néanmoins, elles ont influencé les débats sur l'identité collective dans chacun des deux pays, et gagné ou perdu du terrain à peu près aux mêmes époques.

10 L'importance relative de ces conceptions dans les deux pays apparaît avec clarté non seulement dans les déclarations publiques des acteurs politiques, mais aussi dans les programmes d'histoire et de géographie de l'enseignement primaire, intermédiaire et secondaire. La législation relative aux investissements étrangers et à l'acquisition de la citoyenneté reflète également l'importance donnée à la langue comme critère constitutif de la nation. Comme nous l'avons montré notamment pour le code de la nationalité, le législateur syrien tend à privilégier les Arabes par rapport aux nonArabes tout en modifiant ces privilèges de façon plus ou moins restrictive à travers le temps ${ }^{18}$. 
11 Dans le cas concret de l'Égypte, la période qui suit la fin de la deuxième guerre mondiale et qui correspond aux dernières années de la monarchie est caractérisée par une définition de la nation dont toute référence arabe est absente. Dans le discours des forces dominantes de l'ancien régime, y compris le roi et le Wafd, l'Égypte constitue une nation (umma) en elle-même, délimitée par les frontières de l'État ${ }^{19}$. Certes, elle se veut souvent solidaire des Arabes, mais ceux-ci forment autant de "nations arabes" (umam 'arabiyya) qu'il y a d'États et d'entités politiques arabes ${ }^{20}$. Même la Ligue des États arabes (Jama'at al-duwal al-'arabiyya) est souvent nommée «Ligue des nations arabes » (Jama'atal-umam al-'arabiyya) ${ }^{21}$. Du reste, la solidarité avec les « nations arabes » est souvent évoquée dans le contexte d'une solidarité non moins étroite avec «les peuples et nations musulmans » et « orientaux ${ }^{22}$.

12 Même si les notions arabe de umma et française de "nation » ne sont pas forcément identiques et comportent des connotations différentes, elles servent toutes deux à désigner une population dont les membres sont liés entre eux par une solidarité spécifique, qu'elle soit ou non dérivée de la loyauté envers un individu ou une institution supérieurs. Dans les deux cas, cette solidarité se construit sur la base de traits distinctifs partagés par les membres du groupe et seulement par eux.

Nation et umma peuvent donc être considérées comme des équivalents dans le présent contexte.

14 La révolution de 1952 et le régime des officiers libres, sous Gamal Abdel Nasser, n'affectent pas dans l'immédiat la notion de nation égyptienne. Il faut attendre deux ans pour que le discours officiel commence à insister sur l'arabité de l'Égypte, et ce n'est qu'au début de la deuxième moitié de la décennie que le nationalisme arabe devient définitivement l'idéologie identitaire du régime. Le passage à la notion de nation basée sur la langue est alors consacrée par la Constitution de 1956, qui décrit l'Égypte comme une " partie de la nation arabe » (juz al-umma al-'arabiyya) ${ }^{23}$.

15 Néanmoins, pendant l'époque glorieuse des nationalismes arabes à la fin des années cinquante et dans les années soixante, l'Égypte nassérienne ne se fond jamais complètement dans la nation arabe ni ne se confond avec elle. À partir du moment où l'Égypte est redéfinie comme une partie de la nation arabe, la notion de "peuple égyptien » (al-sha'b al-masri) distinct des autres « peuples arabes » (al-shu'ûb al-'arabiyya) ou du reste «du peuple arabe » (al-sha'b al-'arabi) rassure les Égyptiens quant à leur spécificité. L'Égypte revendique par ailleurs sa centralité au sein de la nation arabe: ainsi arrive-t-il qu'un dirigeant comme le maréchal Amr, dans un lapsus, continue à identifier la nation avec l'Égypte ${ }^{24}$.

16 Le président Sadate qui, en 1970, succède à Gamal Abdel Nasser va rapidement réhabiliter l'Égypte comme entité digne d'une loyauté spécifique. Toutefois, le nouveau président préfère se référer à " l'Égypte » qu'au "peuple égyptien » dont, après tout, il se méfie. D'un point de vue rhétorique, le pays reste une partie de la nation arabe, mais une partie toujours plus distincte et spécifique ${ }^{25}$. Après le traité de paix avec Israël en 1979, la version officielle de l'identité égyptienne se passe encore davantage de références arabes. Plus tard, le retour de l'Égypte au sein de la Ligue arabe sous le président Moubarak n'affaiblira en rien l'idée d'identité égyptienne à l'intérieur de la nation arabe ${ }^{26}$.

17 À la différence de l'Égypte, la Syrie des années quarante est fortement imprégnée des «nationalismes arabes » selon la définition donnée plus haut. Dans les déclarations de 
la majorité des acteurs politiques, la République dite «libérale » issue du mandat fait partie du monde et de la nation arabes ${ }^{27}$. De nombreuses voix de l'époque voient même en leur pays le « berceau du nationalisme arabe $»^{28}$. À y regarder de plus près, pourtant, l'arabité de ce nationalisme était fluctuante. Le nationalisme arabe était souvent et avant tout une forme d'anti-impérialisme dirigé contre les pouvoirs européens qui avaient dominé le Machrek depuis la fin de l'Empire ottoman et qui y avaient installé les sionistes. L'idée de fédérer tous les Arabes faisait, certes, partie de ce nationalisme, mais dans la pratique, les frontières tracées par les autorités mandataires se révélaient assez fortes pour contenir en leur sein les forces d'opposition et de résistance ${ }^{29}$; en établissant des arènes politiques séparées ${ }^{30}$, ces frontières réussissaient non seulement à morceler la lutte anti-impérialiste, mais aussi à créer des liens de solidarité spécifiques parmi les résistants actifs dans chacune des arènes. Le conflit avec les nonArabes l'emportait par conséquent sur l'unité inter-arabe.

Malgré le fort sentiment d'appartenance à la " Nation arabe ", il n'est pas rare que les acteurs politiques de la Syrie des années quarante et du début des années cinquante considèrent l'État syrien et sa population comme une entité bien distincte. Le terme de umma - et donc de "nation" - est alors appliqué à la Syrie dans ses frontières «artificielles» de 1939 qui, en plusieurs étapes, furent imposées par les puissances mandataires. Parfois, les deux notions- nation syrienne et nation arabe - coexistent dans un même discours. Ainsi Shukri al-Quwatli, le premier président de la Syrie indépendante, déclare-t-il, le jour du départ des derniers soldats français en 1946, que "Bilâd al-Sham [la Syrie] est le berceau de l'idée de la 'urûba [l'arabité] et le domicile de ses premiers avocats et martyrs", qui n'arborera "aucun autre drapeau que celui de l'unité arabe ", tout en identifiant l'État syrien non seulement avec le watan mais aussi avec la umma $a^{31}$. Comme le confirment d'autres discours de l'époque, l'identité de la Syrie reste ambiguë et le choix n'est pas encore fait quant à la définition de la nation comme entité géographique et sociologique qui commande la loyauté suprême ${ }^{32}$.

L'idée d'une umma syrienne limitée aux habitants de l'État syrien disparaît au cours de la première moitié des années cinquante. Si le passage à l'idée de nation arabe s'opère grosso modo en même temps qu'en Égypte, il s'en distingue néanmoins de façon nette. D'abord, en Syrie, la notion de nation arabe était plus forte dès le début; ensuite, elle n'y a pas été pas imposée rapidement et "par le haut " comme en Égypte mais s'est renforcée grâce à l'évolution progressive de l'opinion publique. L'appartenance arabe de la Syrie est revendiquée avec la plus grande insistance par les nationalistes "révolutionnaires » qui, alors, commencent à pénétrer dans les sphères du pouvoir. Ceux des nationalistes "révolutionnaires", surtout dans les rangs du Parti populaire syrien, qui défendaient au départ l'idée d'une « Grande Syrie » comprenant le Liban et la Palestine au cours des années cinquante, réaménagent ce projet pour y inclure des parties toujours plus vastes $\mathrm{du}$ monde arabe. Enfin, les nationalistes arabes conservateurs ou libéraux, ne serait-ce que pour des raisons tactiques, finissent par soutenir les nationalistes « révolutionnaires » dans leurs exigences d'unité politique. Le processus culmine avec la dissolution de l'État syrien dans la République Arabe Unie (RAU), formée avec l'Égypte en $1958^{33}$.

20 Malgré le repli sur soi qu'elle provoque à beaucoup d'égards, la sécession de la Syrie (infisâl) et par conséquent la fin de la RAU en 1961 ne conduisent pas à la redécouverte d'un nationalisme explicitement syrien ${ }^{34}$. Bien que modifiés, plus circonspects et moins axés sur l'unité constitutionnelle, les nationalismes arabes continuent d'exercer leur 
monopole identitaire pendant les trois décennies suivantes. Après la prise de pouvoir ba'thiste en 1963, les référents proprement syriens de l'identité collective syrienne, déjà largement épurés, disparaissent presque entièrement. Certes, dans la deuxième moitié des années soixante, le courant « régionaliste » du Ba'th (al-qutriyyûn) domine le parti et le pays ${ }^{35}$. Ses représentants poursuivent une politique vigoureuse de statebuilding limitée à la seule Syrie et n'appellent plus à l'unification de la nation arabe dans un seul État. En même temps, pourtant, ils suppriment la notion encore vaguement présente de " peuple syrien » (al-sha'b al-sûn) au sein de cette nation arabe, au profit de celle, d'un seul " peuple arabe » identique à la nation arabe; les Syriens, désormais, ne forment plus que "le peuple arabe de Syrie" (al-sha'b al-'arabi fi Sûriyya) $\|^{36}$. Contraire aux Égyptiens qui, aux yeux de leurs gouvernants, continuent à former un peuple, les Syriens perdent ce reste de spécificité à l'intérieur de la grande nation arabe.

Bien que le discours ba'thiste ait évolué avec et depuis l'avènement de Hâfiz al-Asad en 1970, la définition de l'identité collective des Syriens et de leur appartenance nationale est restée essentiellement la même. Depuis la fin des années quatre-vingt, pourtant, quelques fissures apparaissent dans cet édifice nationaliste apparemment si solide. Sans mettre en cause l'appartenance de la Syrie et des Syriens à la nation arabe, le régime légitime parfois sa politique en termes d'intérêts spécifiquement syriens. Le plus significatif à cet égard est le discours prononcé par Hâfiz al-Asad au début de son nouveau mandat présidentiel en 1992. Au lieu d'appeler à l'unité de la nation au sens qawmi - et donc arabe - du terme, il justifie les divergences politiques entre États arabes et consacre ensuite les deux tiers de son allocution à la nécessité d'oeuvrer pour l'unité - au sens watani du terme - de la Syrie ${ }^{37}$. Implicitement, donc, le régime réaffirme et même renforce l'idée d'une entité syrienne et d'une solidarité entre Syriens à l'intérieur de la nation arabe. Avec bien du retard sur l'Égypte et de façon beaucoup plus modérée que dans l'Égypte sadatienne, une conception concentrique d'identités qawmi et watani voit peu à peu le jour en Syrie.

En résumé, le nationalisme au sens plus étroit ou watani a généralement été beaucoup plus fort en Égypte qu'en Syrie pendant la période qui a suivi la deuxième guerre mondiale. Au début, la notion de watani amême été prééminente en Égypte, tandis qu'en Syrie, c'est toujours la notion plus large (qawmi) qui a dominé. Quand cette dernière gagnait du terrain en Egypte, elle ne remplaçait jamais entièrement la première. En Syrie, par contre, la conception qawmi aréussi pendant longtemps à effacer entièrement la conception watani. Dans les deux pays, la prédominance de la notion plus large - donc de la langue comme critère constitutif de la nation - s'établit au cours des années cinquante; les notions de solidarité collective basées sur des critères autres que la langue sont réapparues en Égypte à partir des années soixantedix, et en Syrie à la fin des années quatre-vingt. Sans avoir retrouvé son impact d'antan, la conception watani est redevenue très importante en Égypte, peut-être même dominante, bien qu'officiellement enrobée dans un discours qui garde encore des apparences qawmi. Et si, en Syrie, le retour au watani n'est encore que timide, il est cependant perceptible. 


\section{Nations flexibles et langue}

23 Comme on vient de le voir, en Syrie et en Égypte, la nation définie comme une communauté supposée recevoir la solidarité suprême de ses membres a changé de frontières depuis la fin de la deuxième guerre mondiale et risque d'en changer encore à l'avenir. Dans les deux pays, la notion dominante de nation devient progressivement plus englobante; puis cette notion englobante, tout en étant maintenue, se trouve érodée à divers degrés par la réhabilitation d'identités définies de façon plus étroite. Ces mouvements d'expansion et de contraction font de la communauté de solidarité une entité flexible dont il faut expliquer les variations. Si, selon les termes de Benedict Anderson, les nations sont avant tout des " communautés imaginées $»^{38}$, elles sont aussi des communautés perpétuellement réimaginées. Lorsque l'acte suit le verbe, ces communautés peuvent même être réaménagées et reconstruites à travers des politiques qui étayent l'imagination, par exemple par la modification de la loi sur la nationalité ou par la fusion d'États existants.

Les processus à travers lesquels sont redessinées les frontières d'une nation ou communauté de solidarité ne peuvent évidemment être expliqués par rapport à une seule variable indépendante. Même la multiplicité d'intérêts pouvant être invoquée pour justifier une approche instrumentaliste comme la nôtre n'épuise pas nécessairement la multiplicité des facteurs à l'œuvre. Il ne peut donc s'agir ici que d'explorer le rôle qu'ont joué certains intérêts dans les processus en question, et non de les expliquer pleinement.

Observons tout d'abord que l'«arabisation » de la notion de la nation en Syrie et en Égypte coïncide chronologiquement avec une forte perception de menace extérieure. De même, les tendances vers la « désarabisation » de l'identité collective dans les deux pays, aussi inégale leur importance soit-elle, coïncident avec l'affaiblissement de ces menaces extérieures aux yeux des principaux producteurs du discours politique.

En Égypte, le régime nassérien qui, au milieu des années cinquante, domine le discours politique sans encore le monopoliser, met alors l'accent sur les éléments arabes de l'identité égyptienne parce qu'il cherche à mobiliser les Arabes contre les pactes militaires anti-soviétiques proposés par la Grande-Bretagne et les États-Unis. L'« arabisation » rhétorique de l'Égypte doit servir à rallier les Arabes à la cause égyptienne; plus précisément, elle doit contribuer à contrer ce qui apparaît comme une menace de domination néo-coloniale et, en même temps, à renforcer la position du Caire dans la région. Plus s'enveniment les débats et conflits interarabes autour de la « défense du Moyen-Orient » et du pacte de Bagdad, plus l'Égypte se présente comme un pays et une force arabes. Le processus culmine avec la nationalisation du canal de Suez et l'agression tripartite contre l'Égypte en $1956^{39}$. La nécessité de mobiliser le monde arabe pour sortir victorieux de cette crise boucle le passage du nationalisme égyptien au nationalisme arabe. L'insistance sur la nouvelle identité arabe de l'Égypte est surtout destinée à l'extérieur: cela apparaît clairement dans les précautions rhétoriques du régime, qui continue d'accepter l'existence d'un "peuple égyptien » à l'intérieur de «la nation arabe».

27 De façon analogue, la « désarabisation " partielle de l'identité égyptienne à partir des années soixante-dix reflète les choix politiques d'Anouar al-Sadate qui, tout en cherchant le soutien militaire et économique des Arabes, refuse d'être entraîné dans des conflits qu'il juge incompatibles avec la reconstruction et le développement du pays 
après la guerre de $1967^{40}$. Le nouveau ra'is tire ainsi la leçon de l'erreur de son prédécesseur, qui ne voulut point mener cette guerre contre Israël mais s'y sentit contraint pour sauver sa légitimité dans le monde arabe. Si la défaite ne détruit pas entièrement cette dernière, elle délégitimise pourtant aux yeux de beaucoup d'Égyptiens, dont Anouar al-Sadate lui-même, le nationalisme arabe sous la bannière duquel fut menée la guerre. Une fois au pouvoir, Sadate entend donc fermement faire "sa " guerre, conçue comme la dernière, au moment choisi par lui-même, ce qu'il réussit en 1973 avec la coopération de la Syrie. Afin d'obtenir le soutien nécessaire pour repousser Israël et pour, ensuite, réussir sa politique de libéralisation économique, le président Sadate doit sans doute courtiser donateurs et investisseurs arabes, mais pas au point d'effacer l'identité égyptienne.

28 En Syrie, le passage définitif au nationalisme arabe comme idéologie identitaire dominante coïncide, comme en Égypte, avec les projets de défense occidentaux issus de la guerre froide. Davantage encore qu'en Égypte, ces projets sont considérés en Syrie comme des menaces pour l'indépendance fraîchement acquise du pays. Pourtant en Syrie, à la différence de l'Egypte, cette perception trouve pendant longtemps son expression spontanée à tous les niveaux d'un système politique encore pluraliste. Sous le mandat, l'ingérence française dans les affaires du pays était plus directe et importante que celle de l'Angleterre en Égypte. De plus, la Syrie est de loin plus petite, sa population moins nombreuse, sa stabilité étatique précaire. Enfin, Israël, considéré comme une création et une tête de pont impérialistes, est plus proche de la Syrie que de l'Égypte; dès 1951, l'incident du lac Hula illustre les dangers pouvant venir d'une terre qui, dans un passé pas si éloigné fut encore appelée, avec le reste de la Palestine, la «Syrie du Sud » (Sûriyya janûbiyya) ${ }^{41}$.

Deux tentatives de coup d'État contre le gouvernement démocratiquement élu de la Syrie, la première en 1956 et la deuxième en 1957, renforcent le sentiment d'une menace venant des forces impérialistes. Les puissances occidentales sont en effet fortement impliquées dans les deux complots. Lors de la conspiration de 1957, la Turquie, qui a signé le pacte de Bagdad et qui, de plus, est membre de l'OTAN, concentre des troupes sur la frontière syrienne. Simultanément, la doctrine Eisenhower, énoncée la même année, peut faire craindre une intervention militaire américaine pour " protéger » le pays contre le communisme. Le danger paraît d'autant plus grand qu'aux États-Unis, les nationalistes arabes sont assimilés de façon croissante aux communistes. On prévoit une majorité de voix pour les divers partis nationalistes arabes et les communistes aux élections de 1958. Il est presque logique, dans ces conditions, de craindre un coup d'État militaire de la part de forces conservatrices qui, par la suite, pourraient invoquer la doctrine Eisenhower ${ }^{42}$.

30 À partir des années soixante, notamment après la prise du pouvoir par les ba'thistes en 1963, l'analyse des menaces extérieures devient plus complexe. D'une part, nombre de ba'thistes et, parmi eux, surtout les plus jeunes, considèrent Israël comme une menace plus grande qu'il ne l'était aux yeux de leur prédécesseurs. Par conséquent, leur attitude envers l'État hébreu est plus hostile. D'autre part, après la sécession de la Syrie qui a mis fin à la RAU, les dirigeants du pays se sentent obligés de prouver leur dévouement à la cause arabe, non seulement pour contrer les attaques extérieures de Nasser mais aussi pour défendre leur légitimité à l'intérieur. Ainsi apparaissent de nouvelles menaces qui, elles aussi, renforcent l'insistance sur l'identité arabe du pays et du régime. 
Or, la recherche de légitimité intérieure n'est pas facile à concilier avec la recherche d'un soutien de Nasser : car pour acquérir la première, il s'agit de prouver que le Bath est un meilleur défenseur que Nasser de la cause arabe. Si la recherche du soutien de Nasser pousse à une attitude "nationaliste" vis-à-vis d'Israël, la recherche de légitimité intérieure pousse à la surenchère. C'est cette politique de surenchère qui exacerbe le conflit entre la Syrie et Israël et qui menace la légitimité arabe de Nasser au point où, en 1967, il tombe dans le piège d'une logique de guerre. Bien qu'agacé par les ba'thistes syriens, Nasser ne peut que leur accorder son soutien.

Par ailleurs, la radicalisation de la politique de Damas vis-à-vis d'Israël renforce encore davantage le processus d'«arabisation » de l'identité collective de la Syrie ; seule cette arabisation paraît en mesure de mobiliser les ressources nécessaires pour affronter l'hostilité grandissante d'Israël envers la radicalisation syrienne. Le jeu se solde pourtant par la défaite cuisante de 1967, qui enlève le Golan à la Syrie comme elle enlève le Sinaï à l'Égypte. La Syrie est plus vulnérable que jamais. Dans cette situation, ses dirigeants, plus que ceux de l'Égypte, continuent à miser sur le soutien arabe et, à cette fin, sur l'arabité de leur identité.

Malgré leurs différences, donc, les cas de l'Égypte et de la Syrie montrent clairement une coïncidence chronologique entre les perceptions de menaces extérieures, d'une part, et la définition de la nation ou de l'identité collective, d'autre part. Lorsque les dangers augmentent, les frontières de la communauté de solidarité tendent à se dilater; lorsqu'au contraire ils diminuent, l'identité collective est redéfinie de façon plus restrictive et spécifique.

En Syrie, les menaces extérieures résolvent une ambiguïté identitaire initiale qui oppose syro-centrisme et arabisme. Seul le rapprochement et l'identification, puis la fusion de la Syrie avec le reste du monde arabe paraît lui donner une assurance de sécurité contre les menaces extérieures, y compris celles venant de la monarchie irakienne d'alors, assimilée aux forces impérialistes et anti-arabes. En devenant "pleinement arabe ", la Syrie cherche à profiter du soutien automatique des Arabes non syriens, espérant qu'ils ne feront pas de différence entre eux-mêmes et la Syrie. Plus tard, à la fin des années quatre-vingt, l'État syrien ne vit certes pas en paix avec ses voisins mais se trouve cependant moins menacé. Son emprise sur le Liban est ferme, et si le Golan se trouve toujours sous occupation israélienne, le statu quo avec Tel-Aviv ne risque pas de se détériorer davantage. Il est donc possible de réhabiliter quelques éléments d'une identité plus spécifiquement syrienne.

En Égypte, le passage au nationalisme arabe à partir de 1954-55 sert également, et de façon bien plus évidente, à mobiliser tous les Arabes pour défendre les intérêts de certains d'entre eux - ou plutôt, de ceux qui, pour l'occasion, se sont convertis à l'arabisme. Plus confiants dans leur force propre et celle de leur pays, les dirigeants égyptiens, contrairement à leurs contemporains syriens, ne sont pourtant pas tentés ou obligés d'effacer toute différence entre leur « peuple » et sa nouvelle «nation ». Quand les dangers sont surmontés et surtout, quand la politique de solidarité et d'unité arabe choisie pour les affronter devient contre-productive dans la crise de 1967 et, en effet, mène au désastre, les mêmes considérations de sécurité imposent un repli sur une identité plus spécifiquement égyptienne.

Les dirigeants égyptiens, plus tôt que les Syriens, avaient reconnu que l'élargissement de la nation était une épée à double tranchant. Elargir la nation sans contrôler ceux qui la rejoignent ou ceux que l'on rejoint peut générer des inconvénients qui annihilent les 
avantages. Dans un sens, l'expérience de la RAU l'avait montré aux Syriens qui, tout en restant nationalistes arabes, évitaient depuis lors les arrangements constitutionnels contraignants. En termes généraux, l'élargissement de la nation permet, certes, de mobiliser des ressources supplémentaires, mais comporte également le risque de perdre des ressources auparavant moins contestées. Au non de l'identité nationale désormais partagée, des forces jadis extérieures ou étrangères peuvent ainsi s'ingérer dans les affaires «intérieures ». Une Syrie arabe et nationaliste arabe peut trouver un soutien en Égypte et en Irak, mais elle s'expose également aux tentatives des nassériens égyptiens et des ba'thistes irakiens qui cherchent à mobiliser, en Syrie, le soutien à leurs propres causes.

En dehors de toute considération de sécurité, le risque de se voir ainsi couper l'herbe sous le pied n'encourage guère des projets politiques, sociaux ou économiques à long terme. Dans un contexte politique relativement peu institutionnalisé comme c'est le cas au Proche-Orient, les protagonistes de tels projets cherchent donc à recréer des arènes politiques qu'ils peuvent dominer, si la domination n'est pas déjà leur but principal. Or le rétablissement d'arènes politiques maîtrisables nécessite un contrôle de l'accès à ces arènes. Celui-ci se fait plus facilement au moyen de cartes d'identités, et donc d'identités spécifiques, qui reproduisent le découpage entre les arènes.

En Égypte comme en Syrie, l'extension et la contraction de la communauté de solidarité apparaissent donc largement comme la fonction de stratégies et d'intérêts précis. Les considérations de sécurité dominent les périodes d'extension et jouent un rôle important dans les processus de contraction qui, pourtant, obéissent également à une logique d'intérêt économique dans le sens le plus large. En d'autres termes, le déplacement des frontières de la nation permet de poursuivre de nouvelles stratégies politiques.

\section{Intérêt et langue}

En Syrie et en Égypte, le critère choisi à partir des années cinquante pour dessiner les frontières de la nation élargie est celui de la langue. La communauté de solidarité qui doit affronter les menaces de la part de tiers est celle des Arabes définis comme ceux qui parlent l'arabe. Si d'autres options, comme les liens qui peuvent unir la population de la Grande Syrie ou de la vallée du Nil, sont écartées, c'est certainement en partie en raison de la force que la nation arabe avait alors acquise comme idée mobilisatrice et légitimatrice. Cette force, l'idée de nation arabe l'avait acquise non seulement du fait qu'elle définissait une identité collective à partir de la langue, mais qu'elle la définissait à partir d'une langue partagée par des populations pouvant également partager une expérience historique en partie commune.

40 À l'époque des grands dangers des années cinquante, le poids déjà accumulé de cette idée de nation arabe seule et unie explique pourtant moins son triomphe que le simple potentiel de mobilisation inhérent à la langue comme moyen de communication par excellence. Vu l'étendue de l'aire géographique où l'on parle l'arabe, ce potentiel de mobilisation paraissait impressionnant. Grâce à l'homogénéité de la langue écrite, la portée identitaire des différences locales et régionales de la langue parlée pouvait être réduite avec une relative facilité. Plus efficacement qu'en d'autres cas, le potentiel de mobilisation de la langue pouvait donc être traduit en réalité. Inversement, aucun 
autre critère, y compris religieux, n'aurait permis à l'époque d'imaginer ou de constituer une communauté de cette taille apte à relever le défi extérieur.

Si la langue était particulièrement adaptée à créer ce que K. W. Deutsch aurait appelé une "communauté de défense " ${ }^{43}$, elle était également bien adaptée à ronger les cloisons entre les diverses arènes politiques que la plupart des acteurs politiques arabes cherchaient à maintenir ou à reconstruire pour leur propre profit. Davantage que d'autres traits communs, la langue crée un lien qui invite aux désertions ou aux ingérences dans les affaires «intérieures » et ainsi, menace les pouvoirs en place ou leur projets politiques. Seule la relativisation de la continuité linguistique par des discontinuités diverses, y compris non linguistiques, permet alors de défendre ces intérêts.

\section{NOTES}

1. Parmi les rares exceptions qui confirment la règle, mentionnons CARRÉ O., Le nationalisme arabe, Fayard, Paris, 1993, et l'atelierde recherche Rethinking Nationalisms inthe Arab World réuni par J. Jankowski et I. Gershoni du 22 au 24/9/1994 à l'Université du Colorado (Boulder), dont les travaux ne sont pas encore publiés.

2. Ainsi KEPEL G., Le Prophète et Pharaon: les mouvements islamistes dansl'Égypte contemporaine, La Découverte, Paris, 1984; 0. Carré et P. Dumont (dir.), Radicalismes islamiques, 2 tomes, L'Harmattan, Paris, 1986 ; AL-AZMEH A., Islams and Modernities, Londres,1993 ; AUBI N., Political Islam : Religion and Politics in the Arab World, Routledge, Londres, 1991.

3. Cf. SEALE P., The Struggle for Syria: A Study of Post-War Arab Politics 1945-1958, nouvelle édition, I.

B. Tauris, Londres, 1986.

4. Sur le ba'thisme, son évolution et ses variantes, voir par exemple CARRÉ O., 1993, op. cit., p. 39-70 ; CARRÉ 0., «Le mouvement idéologique ba'thiste ", La Syrie aujourd'hui (A. Raymond, dir.), Editions du CNRS, Paris, 1980, p. 185-206; ABU JABIR K. S., The Bath Party: History, Ideology and Organization, Syracuse, Syracuse University Press,N. Y, 1966, ainsi que les titres mentionnés plus bas.

5. Cf. LAURENS H., Le grand jeu: Orient arabe et rivalités internationales depuis1945, Armand Colin, Paris, 1991, p. 25 ; P. Seale, 1986, op. cit., surtout les chapitres 2,3,6, 16 et 17.

6. Sur l'évolution du nassérisme (du nationalisme égyptien au nationalisme arabe), voir NASR M., AI-tasawwur al-qawmi al-'arabi fi fikr Gamal 'Abd al-Nâsir, 1952-1970, Markazdirâsât al-nahda al-'arabiyya, Beyrouth, 1981; CARRÉ 0., 1993, op. cit. p. 91-154; sur l'idéologie nassérienne en matière de politique socio-économique et de politique étrangère, voir ROUSSILLON A., «L'Égypte républicaine : trois régimes pour une révolution ", Histoire de l'Égypte de la conquête arabe à nos jours (G. Alleaume et H. Laurens dir.), Paris, à paraître ; WATERBURY J., The Egypt of Nasser and Sadat: The Political Economy of Two Regimes, Princeton, Princeton University Press, N. J., 1983 ; pour une comparaison brève mais pertinente entre nassérisme et ba'thisme, voir LAURENS L, 1991, op. cit., p. 159.

7. Ainsi DEVLIN J. F., The Baih Party: A History from its Origins to 1966, HooverInstitution Press, Stanford, Ca., 1976; PICARD E., «La Syrie de 1946 à 1979 », La Syrie aujourd'hui, op. cit.; RABINOVITCH I., Syria under the Baih 1963-1966: The Army-PartySymbiosis, Israël University Press, New York Tel Aviv, 1972. 
8. Pour cette période, voir surtout DAWN C. E., From Ottomanism to Arabism : Essays on the Origin of Arab Nationalism, Urbana, University of Illinois Press, III., 1973 ; HOURANI A., Arabic Thought in the Liberal Age 1798-1939, Cambridge University Press, Cambridge, 1983, p. 193-221 ; KHOURY P., Urban Notables and Arab Nationalism: The Politics of Damascus 1800-1920, Cambridge University Press, Cambridge, 1983 ; TIBI B., Arab Nationalism : A Critical Enquiry, St Martin's Press, New York, N.Y, 1981.

9. Cf. NASR, op. cit., 1981 ; sur la notion de nation spécifiquement égyptienne et les nationalismes égyptiens, voir JANKOWSKI J. et GERSHONI I., Egypt, Islam and the Arabs: The Search for Egyptian Nationhood, Oxford University Press, New York, N.Y, 1986 ; ROUSSILLON A., « Egyptianité, arabité, islamité : la recomposition des référents identitaires ", Égypte/Monde arabe 11, 3e trim. 1992, p. 77-135 ; STEPPAT F., « Nationalismus und Islam bei Mustafa Kamil », Welt des Islam, tome IV, 1956, p. 241-341.

10. SEALE P., op. cit., 1986, chapitre 8 ; YAMAK L. Z., The Syrian Social Nationalist Party: An Ideological Analysis, Cambridge, Mass., 1966.

11. VAN DUSEN M. H., «Political Integration and Regionalism in Syria », Middle East Journal, tome XXVI, n², p. 123-136 ; SEURAT M., «Les populations, l'État et la société », La Syrie aujourd'hui, op. cit., p. 87-141.

12. Voir ESMAN M. et RABINOVITCH L., Ethnicity, Pluralism and the State in the Middle East, Ithaca Press, New York, 1988 ; SCHEFFLER T., Ethnizitat und Gewalt, Deutsches Orient-Institut, Hamburg, 1991.

13. Une exception récente qui, pourtant, ne néglige pas l'importance des divisions sociétales verticales: HANF T., Koexistenz im Krieg: Staatsverfall und Entstehung einer Nation im Libanon, Nomos, Baden-Baden, 1990.

14. Parmi les "classiques ", on notera BATATU H., The Old Social Classes and the Revolutionary Movements of Iraq, Princeton University Press, Princeton, N. J., 1978, et HUSSEIN M., Égypte, lutte de classes et libération nationale, 1967-1973, Maspéro, Paris, 1975 ; parmi les ouvrages plus récents : SADIQ M., Hiwar hawla Sûriya, Le Caire, 1993.

15. Une des rares monographies consacrées à cette thématique reste BARAM A., Culture, History and Ideology in the Formation of Ba'thist Iraq 1968-1989, Basingstoke Macmillan, Londres, 1991.

16. Notre conception du nationalisme s'inspire des approches subjectivistes et instrumentalistes proposées par exemple par HOBSBAWM E., Nations and Nationalism since 1780 : Programme, Myth, Reality, Cambridge University Press, Cambridge, 1990 ; GELLNER E., Nations and Nationalism, Basil Blackwell, Oxford, 1983, ou ANDERSON B., Imagined Communities: Reflections on the Origin and Spread of Nationalism, Verso, Londres, New York, N. Y., 1983 ; ainsi que, de façon implicite, par NOIRIEL G., "La question nationale comme objet de l'histoire sociale », Genèses 4, mai 1991, notamment p. $86 \mathrm{~s}$.

17. Cf. le célèbre débat entre Tawfîq al-Hakîm (al-Ahrâm, 03 et 12/03/78; Al-Akhbâr, 18/03/78), Husayn Fawzi (Al-Akhbâr 23/03/78) et Louis 'Awad (Al-Ahrâm, 07/04/78), d'une part, et Wahîd Rif'at (al-Akhbâr, 11 et 25/03/78), d'autre part. Plus récemment, voir SAYYID SA'ÎD M., Mustaqbal al-nizâm al-'arabi ba'd azmat al-khalij (L'avenir du système arabe après la crise du Golfe), Al-majlis al-watani li-l-thaqâfa wa-l-funûn wa-l-adab, Koweit, 1992; dans un contexte où a été introduite une notion de «moyen-orientalisme » (al-sharq al-awsatiyya) qui inclut Israël, le débat, bien que modifié, continue, comme par exemple dans Al-mutamar al-qawmial-islâmi al-awwal (Premier congrès national islamique), Centre d'études de l'unité arabe, Beyrouth, 1995.

18. KIENLE E., « Imagined Communities Legislated : Nationalisms and Law of Nationality in Syria and Egypt ", Yearbook of lslamic and Middle Eastem Law 1994, Cotran E., Mallat C. eds, Kluwer Law International, Londres, 1995, p. 47-67.

19. Voir par exemple la déclaration de politique générale de Mahmûd Fahmi Nuqrashi, alors premier ministre, devant la Chambre des députés le 15/12/47, Al-Ahrâm, 16/12/47 ou celle d'un de ses successeurs, Ismâ'îl Sirri, le 01/08/49, al-Ahrâm, 02/08/49. 
20. Cf. Nuqrashi dans sa déclaration célébrant la fin de la deuxième guerre mondiale, Bilâdi, 18/05/45 ; également le roi Farûq dans son discours du trône (Khitâb al-'arsh) du 16/01/50, AlAhrâm, 17/01/50.

21. Ainsi dans un débat du Sénat le 12/03/45, Al-Ahrâm, 13/03/45.

22. Discours du trône le $16 / 01 / 50$, cf. supra.

23. Al-Ahrâm, $16-18 / 01 / 56$ et $23-24 / 06 / 56$; traduction du texte en français : J. E. Godchot, Les constitutions du Proche et du Moyen-Orient, Sirey, Paris, 1957, p. 45 s. ; voir également NASR, op. cit., 1981, en particulier p. 107 et 125.

24. Voir, par exemple, President Gamal 'Abd al-Hasir's Speeches and Press Interviews during the Year 1958, United Arab Republic, Ministry of Information, Le Caire, s. d., p. 4 s. ; discours de Nasser le 22/07/63, al-Ahrâm, 23/07/63 ; discours du maréchal Amr le 07/11/63, al-Ahrâm, 08/11/63 ; voir également Nasr, 1981, p. 383, $396 \mathrm{~s}$.

25. Voir, par exemple, Speeches by President Anwar al-Sadat, September 1970-March 1971, United Arab Republic, Ministry of Information, Le Caire, s. d., p. 1 s., 114.

26. Cf. discours du président Moubarak prononcés le 25/04/92 et le 22/07/92, al-Ahrâm, $26 / 04 / 92 ; 23 / 07 / 92$.

27. Cf. la résolution principale du congrès fondateur du Parti national (al-hizb al-watani), avril 1947, The Arab States and the Arab League:A Documentary Record, M. Khalîl (dir.), Khayyat, Beyrouth, 1962, tome 1, p. 670 s., et AI-Ahzab al-siyasiyya fi Sûriyya (anonyme), Damas, 1954, p. 192-205 ; cf. également le communiqué du Parti du peuple (hizb al-sha'b) du 15/08/48, AlAhzab..., p. 157-170.

28. Parmi eux, le premier président de la Syrie indépendante, Shukri al-Quwatli, lors de son discours à l'occasion du départ du dernier soldat français le 17/04/46, Yawm al-qawmi al-'arisa : Jala' al-juyush al-ajnabiyya 'an Sûriyya, Jarfdat al-Barada (dir.), Damas, 1946, p. 1-13.

29. Voir par exemple KHOURY P., Syria and the French Mandate: The Politics of Arab Nationalism 1920-1945,1. B. Tauris, Londres, 1987.

30. Cf. la notion de "national political field» de S. ZUBAIDA, Islam, the People and the State, Routledge, Londres, 1989, p. 126, 162.

31. Jarîdat al-Barada, op. cit.

32. Cf. Khâlid al-'Azm, alors ministre des Affaires étrangères, lors du débat de l'Assemblée consacré au projet de la "Grande Syrie » proposé par le roi 'Abd Allah de Transjordanie; voir aussi les procès-verbaux de la séance du 23/11/46, Al-Mashâri' al-wahdawiyya al-'arabiyya 1913-1987, Y. Khouri (dir.), Markaz dirâsât al-wahda al-'arabiyya, Beyrouth, 1988, p. 115 s.

33. Cf. SEALE P., 1986, op. cit.; AL-SAYYID M. K., " The Rise and Fall of the United Arab Republic ", 17e colloque du Centre d'études arabes, Université de Georgetown, Washington D. C., avril 1992 (manuscrit).

34. Cf. la proclamation $n^{\circ} 1$ des sécessionnistes en 1961, Al-Mashâri'..., op. cit., 1988, p. 371.

35. Voir DEVLIN J., 1976, et PICARD E., 1980, op. cit..

36. Par exemple, Salah al-Dîn Bitar, alors ministre des Affaires étrangères, le 11/03/63, Alwathâ'iq al-'arabiyya, tome 1, Université américaine de Beyrouth, 1963, p.51; communiqué du régime en date du 23/02/66, Al-wathâ'iq..., tome 4, Beyrouth, 1966, p. $117 \mathrm{~s}$.

37. Al-Bath, 12/03/92.

38. ANDERSON B., op. cit., 1983.

39. Sur la chronologie des événements, voir notamment DAWISHA A. I., Egypt in the Arab World: the Elements of Foreign Policy, Macmillan, Londres, 1976 ; LAURENS H., 1991, op. cit., p. 141-155 ; KERR M. H., The Arab Cold War : Gamal Abd al-Nasir and his Rivais 1958-1970, Oxford University Press, Londres, Oxford, 1971 ; pour une réévaluation des événements, voir Suez 1956: The Crisis and its Consequences, R. Owen et R. W. Louis (dir.), Oxford, 1989.

40. Voir, par exemple.WATERBURY J., op. cit., 1983. 
41. KHOURY P., "Factionalism among Syrian Nationalists during the French Mandate", International Journal of Middle East Studies, vol. XIII, $\mathrm{n}^{\circ} 4,1981$, p. 441-469.

42. Sur la vulnérabilité de la Syrie, voir KERR M. H., 1971, op. cit. ; LAURENS H., 1991, chapitre 4 ; RATHMELL A., Secret War in the Middle East: The Covert Struggle for Syria, 1949-1961, I. B. Tauris, Londres, 1995 ; SEALE P., 1986, op. cit., chapitre 22.

43. DEUTSCH K. W., The Analysis of International Relations, Englewood Cliffs, Prentice-Hall, N. Y., $2^{\mathrm{e}}$ éd., 1978, p. 241-251.

INDEX

Mots-clés : arabe (langue), nationalisme, Syrie

\section{AUTEUR}

\section{EBERHARD KIENLE}

SOAS (Londres)/Cedej 